\title{
Individual Differences in Eye-Movements During Reading: Working Memory and Speed-of-Processing Effects
}

\author{
Matthew J. Traxler \\ University of California Davis \\ Debra L. Long \\ University of California Davis \\ Kristen M. Tooley \\ University of California Davis
}

\author{
Clinton L. Johns \\ University of California Davis \\ Megan Zirnstein \\ University of California Davis \\ Eunike Jonathan \\ University of California Davis
}

\begin{abstract}
Theories of eye-movement control in reading should ultimately describe how differences in knowledge and cognitive abilities affect reading and comprehension. Current mathematical models of eye-movement control do not yet incorporate individual differences as a source of variation in reading, although developmental and groupdifference effects have been studied. These models nonetheless provide an excellent foundation for describing and explaining how and why patterns of eye-movements differ across readers (e.g., Rayner, Chace, \& Ashby, 2006). Our focus in this article is on two aspects of individual variation: global processing speed (e.g., Salthouse, 1996) and working-memory capacity (e.g., Just \& Carpenter, 1992). Using Hierarchical Linear Modeling (HLM) (Raudenbush \& Bryk, 2001), we tested the extent to which overall reading speed and working-memory capacity moderate the degree to which syntactic and semantic information affect fixation times. Previous published data (Traxler et al., 2005) showed that working memory capacity and syntactic complexity interacted to determine fixation times in an eye-movement monitoring experiment. In a new set of models based on this same data set, we found that working-memory capacity interacted with sentencecharacteristic variables only when processing speed was not included in the model. We interpret these findings with respect to current accounts of sentence processing and suggest how they might be incorporated into eye-movement control models.
\end{abstract}

Keywords: Eye-movements, reading, individual differences, working memory

1 


\section{Introduction}

A complete account of reading should include descriptions and explanations of how individual readers differ from one another in ways that give rise to different patterns of eye-movements. In general, skilled readers show considerable similarity in their eye-movementsthey have mostly forward saccades, fixate words for 250 milliseconds or so on average, and so on. The extent to which their patterns of eye-movements differ, however, has received less attention than other aspects of reading (although see Ashby, Rayner, \& Clifton, 2005; Kemper, Crow, \& Kemtes, 2004; Kliegl, Grabner, Rolfs, \& Engbert, 2004; and Rayner, Reichle, Stroud, Williams, \& Pollatsek, 2006, for recent studies assessing age-related and skill-related differences in eye-movements during reading). Thus, we have good accounts of how lexical and sentence-level characteristics influence fixation durations, saccade length, and word skipping, but we lack formal models that specify how differences in various cognitive capacities moderate the effects of text variables on patterns of eye-movements. Our goal in this article is to begin sketching out such an account and to demonstrate how multi-level modeling (also known as Hierarchical Linear Modeling or Mixed Effect modeling) can be used to examine the interactions among individual and text characteristics and their influence on eyemovement patterns during reading. Fortunately, a number of specific formal models of eye-movement control (e.g., E-Z Reader, Reichle, Pollatsek, Fisher, \& Rayner, 1998; and SWIFT, Engbert, Nuthmann, Richter, \& Kliegl, 2005; Engbert, Longtin, \& Kliegl, 2002) provide a principled basis for predicting how and why patterns of eye-movements might differ across readers.

Before embarking on a full investigation of individual differences in reading, we should consider whether the null hypothesis might be true, at least for some aspects of reading behavior. Mature readers may not differ substantially from one another because reading is a highly practiced skill that evolves into a systematic, essentially reflexive behavior. Thus, we might expect overall patterns of eye-movements to be fairly similar across readers with normal levels of intelligence and similar educational backgrounds. Indeed, some studies of reading in old and young adults suggest only a general slowing of reading with increases in age (Kliegl et al., 2004). Other studies suggest that younger and older readers differ in terms of the strategies that they employ during reading, but that the basic cognitive processes involved in lexical access and saccade planning are essentially the same across age groups (Rayner et al., 2006). In contrast, some studies have shown skill-related differences in word-skipping behavior, as well as potential differences in word frequency effects (Ashby et al., 2005). Thus, substantial differences in eyemovement control may remain to be discovered, even among populations with similar age and experience.

Assuming that substantial variation in eyemovements does occur in a population of normal readers, what individual difference variables might give rise to this variation? Reading comprehension involves language specific processes as well as domain-general cognitive abilities - perception, attention, memory, and reasoning. Variation in any of these abilities potentially underlies individual differences in reading behavior and comprehension performance. Most of the research on individual-differences in reading has focused on language-specific factors, like the quality of readers' lexical representations (as in Perfetti's verbal efficiency theory, 1985) or on variation in domain-general cognitive factors (see Long, Johns, \& Morris, 2007, for a review). For example, differences in comprehension ability can be modeled as a function of variation in cognitive control, the ability to flexibly allocate attention, or the ability to suppress irrelevant information (e.g., Gernsbacher, 1989, 1993; Gernsbacher \& Faust, 1991; cf. Engbert et al., 2002); and these factors could be incorporated into models of eye-movement control.

Working-memory capacity is another domaingeneral cognitive ability that has received considerable 
attention as a potential explanation of individual variation in language comprehension and processing efficiency (e.g., Just \& Carpenter, 1992; see also Baddeley \& Hitch, 1974). The general claim is that individuals differ in their ability to maintain information in an active state and to perform operations on that information. If working-memory resources are overtaxed, processes that should be executed simultaneously must be reorganized to run sequentially, or information will be lost and processing will be disrupted. In reading, working-memory capacity must be allocated to the various processes involved in activating lexical representations and in integrating them into developing syntactic and discourse representations. Presumably, individuals who have large workingmemory capacities can execute more processes in a given period of time than individual with small capacities; thus, they should also have shorter fixation durations. Moreover, readers with large capacities should be less affected by manipulations that increase complexity at various levels of representation than readers with small capacities. The account of described above assumes that reading and language interpretation operations are not fully automated and that tests of working- memory capacity tap the same resources as those used in reading and comprehension (see, e.g., Caplan \& Waters, 1990, 1999; MacDonald \& Christiansen, 2002; Waters \& Caplan, 1996).

The exact influence of working memory capacity on language comprehension processes remains controversial (Traxler \& Long, in press; Fedorenko et al., 2006, 2007). Thus, it is important to determine the exact relation between working-memory resources and reading before working-memory differences are incorporated in models of eye-movement control. Sentence processing studies involving eye-tracking and related experimental methods have been used to investigate how working memory differences contribute to differences in sentence processing performance (e.g., King \& Just, 1991; Clifton et al., 2003). In the sentence-processing literature, working-memory effects on language interpretation processes have been investigated in the context of syntactic complexity manipulations. The logic is that working-memory limitations may place an upper bound on how many language processing operations can occur simultaneously and that sentences with complex syntactic structures place substantial demands on working memory. Thus, complexity effects should increase as working-memory resources are reduced, either by means of an external working-memory load or by intrinsic differences among readers. Such interactive effects are commonly observed in studies involving dual-task paradigms in which participants both read and execute a secondary task simultaneously, such as rehearsing a list of unrelated words that they must recall later (e.g., Wanner \& Maratsos, 1978). Interactions of workingmemory capacity and syntactic complexity are sometimes reported in eye-movement and self-paced reading studies as well (e.g., King \& Just, 1991; but cf. Waters \& Caplan, 2003), but sometimes they are not (Clifton et al., 2003; Traxler et al., 2005).

There are a number of reasons why the literature on the relation between working memory and sentence processing includes discrepant findings. First, some studies have used quasi-experimental or extreme-groups designs; these designs have problems of analysis and interpretation that have raised substantial concerns (see Preacher, Rucker, MacCallum, \& Nicewander, 2005). Second, working memory-capacity correlates with many other reader characteristics (e.g., vocabulary, reading speed, print exposure). These other characteristics may account for the variance in participants' reading behavior as well as (or better) than working memory capacity. This raises the possibility that the influence of workingmemory capacity on reading is derivative rather than causal. That is, working-memory capacity shares variation with some other ability and it is this other ability that is responsible for individual differences in sentence processing. To determine whether working memory capacity explains unique variance in eyemovements, ideally the effects of working memory capacity would be assessed in the presence of other individual difference variables. 
Working Memory and Syntactic Complexity. Some previous studies of sentences containing subject and object relative clauses have found correlations between working memory capacity and the effects of syntactic complexity manipulations or other sentence characteristics that render them harder to interpret (King \& Just, 1991; Pearlmutter \& MacDonald, 1995; Traxler et a., 2005). Some of these studies have taken advantage of the difference in processing difficulty associated with subject relative and object relative clauses. In subjectrelative sentences like (1a), the subject noun of the sentence is also the subject of the relative clause (e.g., Director is the subject of watched and won). In objectrelative sentences like (1b), the subject noun of the sentence is the object of the verb in the relative clause (e.g., Director is the subject of won but the object of pleased):

(1a) The director that watched the movie won a prize.

(1b) The director that the movie pleased won a prize.

Eye-movement and other studies consistently find that object-relatives are harder to process than subject relatives (see Traxler, Morris, \& Seely, 2002 for a review). The difficulty associated with object relatives, however, is reduced when semantic information helps the reader construct the correct interpretation (e.g., Mak, Vonk, \& Schriefers, 2002). In sentences like (1c) and (1d), the subject of the sentence is inanimate, and the difficulty associated with the object relative clause nearly disappears (i.e., readers have roughly equivalent patterns of fixations across $1 \mathrm{c}$ and $1 \mathrm{~d})$ :

(1c) The movie that pleased the director won a prize.

(1d) The movie that the director watched won a prize.

Some researchers have interpreted the influence of semantic information on syntactic complexity as showing that the object-relative penalty arises from syntactic reanalysis processes rather than working-memory restrictions. Other researchers have argued that working- memory capacity plays a substantial role in the objectrelative penalty despite the influence of semantic information (e.g., Fedorenko, Gibson, \& Rohde, 2006). Indeed, Traxler et al. (2005) examined the role of working-memory capacity in sentences like (1) and found that it predicted the degree to which syntactic complexity and animacy interacted. They interpreted this interaction as showing that the animacy manipulation served to reduce working-memory load.

One major limitation of the Traxler et al. (2005) study, however, is that they included only one individualdifference variable, working-memory capacity, in their analyses. Because working-memory capacity correlates with numerous other variables, it is possible that working-memory capacity may have moderated the effects of the sentence-level variables as a proxy for another, unanalyzed variable, such as processing speed or experience. Overall reading speed is correlated with performance on standardized reading comprehension tests, print exposure, and patterns of reading in self-paced reading studies (see Long et al., 2007, for a review).

In the current study, we considered the possibility that overall processing speed might have played a critical role in the cross-level interaction of working-memory capacity with animacy and syntactic complexity. Fast readers, who read more often than slow readers, will have greater experience and more practice with language. This may make them more sensitive to semantic cues in syntactic analysis either because they know more about the language or because they can utilize these cues more quickly than slow readers (Long \& Prat, 2007; Pearlmutter \& MacDonald,1995). To assess the contributions of working memory capacity and speed, we re-analyzed data from Traxler and colleagues' (2005) study, using hierarchical linear models that included working-memory capacity and reading speed as predictors of two sentence level variables, clause type (subject relative and object relative) and animacy (animate noun phrase, inanimate noun phrase). We considered two possibilities. First, working-memory capacity may be correlated with reading speed, but each 
may make a unique contribution to the influence of the sentence-level variables. Second, the influence of working-memory capacity may become negligible when reading speed is included in the model because the influence of working-memory capacity on the sentencelevel effects is primarily due to shared variance with reading speed.

To preview the findings, working-memory capacity did not account for significant variance in the eye-movement data when reading speed was included in the model.

\section{Methods}

Participants. 91 native English speakers were paid or received course credit for participating in the eyemovement monitoring portion of the study. All had normal vision or wore soft contact lenses.

Stimuli. The stimuli consisted of 24 sets of sentences like 1a-d (repeated here).

(1a) The director that watched the movie won a prize.

(1b) The director that the movie pleased won a prize.

(1c) The movie that pleased the director won a prize.

(1d) The movie that the director watched won a prize.

Sentences like (1a) contain a subject-relative clause. Changing word order, as in (1b), produced a sentence containing an object-relative clause. Versions (1c) and (1d) were created by changing the order of the two critical nouns and served to counterbalance order. The items, therefore, represent four experimental conditions, with relative clause type (subject vs. object) crossed with animacy of the subject of the sentence (which was always opposite to the animacy of the noun in the relative clause). The animate and inanimate nouns and the verbs in the relative clauses were matched across conditions for length and frequency (within each subject animacy condition, the difference between sentences is merely one of word order, so the sentences are perfectly matched for lexical variables in those contrasts). Pre-testing also assured that the versions were equally plausible.

One version of each item was assigned to one of four lists such that no participant saw more than one version of a item and so that equal numbers of subject- and object-relative sentences appeared on each list. Presentation order was randomized separately for each participant. The test sentences were presented along with 24 sentences from a different experiment and 28 filler sentences of various types. Comprehension questions followed ten of the filler items. All participants scored $90 \%$ or better on the comprehension questions.

Sentence-Span Procedure. Participants completed the Turner and Engle (1989) version of the Daneman \& Carpenter (1980) sentence-span test. Participants received sets of sentences to read aloud. Each sentence was followed by an unrelated target word that the participants were to remember. After reading all of the sentences in a set, participants were asked to recall all of the target words in order. The number of sentences and the number of target words in a set increased from two to six as the participant proceeded through the task. Participants saw three sets of the same size before they saw the next larger set. They were initially given three set of two sentences as practice. Span was calculated as the total number of words correctly recalled across all trials (this procedure produces the best reliability for the sentence span test, Waters \& Caplan, 2003). The possible range of scores was 0 to 60 .

Overall Reading Speed Assessment. A measure of overall reading time was calculated for each participant. This was done by summing the total fixation time for each filler sentence. We then computed an average reading time across all filler sentences.

Eye-movement Monitoring Procedure. A Fourward Technologies Gen 6 dual-purkinje eye-tracker monitored participants' eye movements as they read 
sentences like $(2 \mathrm{a}-2 \mathrm{~d})$. The tracker has angular resolution of $10 \mathrm{~min}$ of arc. The tracker monitored only the right eye's gaze location. A PC displayed materials on a VDU approximately seventy $\mathrm{cm}$ from participants' eyes. The display consisted of Borland $\mathrm{C}$ default font with approximately 4 characters per degree of visual angle.

The location of participants' gaze location was sampled every millisecond and the PC software recorded the tracker's output to establish the sequence of eye fixations and their start and finish times. Participants were seated at the eye tracker and used a bite plate and head rests to minimize head movements. After the tracker was aligned and calibrated, the experiment began. Participants pressed a key after reading each sentence. Comprehension questions followed some of the filler sentences and participants responded by pressing a key labeled "yes" or "no." Participants did not receive feedback on their responses. Between each trial, a pattern of boxes appeared on the computer screen along with a cursor that indicated the participants' current gaze location. If the tracker was out of alignment, the experimenter recalibrated it before proceeding with the next trial.

\section{Results}

We computed three eye-movement measures in two scoring regions, the relative-clause region and the main-verb region. The relative clause region started after the relativizer that and ended immediately before the verb in the main clause. The main verb region consisted of the verb in the main clause. Total time was the sum of all fixations in a region. First-pass time was the sum of fixations beginning with the first fixation in a region and ending when the reader's gaze left the region in any direction. Regression path time was the sum of all fixations in a region beginning with the first fixation and ending when the reader's gaze left the region in the direction of the right boundary.
We analyzed the eye-movement measures for the relative-clause and main-verb regions using hierarchical linear modeling (HLM). As in Traxler and colleagues' (2005) model, Level-1 variables consisted of clause type (subject-relative, object-relative), animacy (animate, inanimate) and their interaction. Level-2 (i.e., individual difference) variables consisted of workingmemory capacity (WMC) and overall RT. Traxler and colleagues included WMC in their model as a Level-2 variable, but not overall RT. The level-1 variables were modeled as fixed effects and the level-2 variables were modeled as random effects. WMC and overall RT were reliably correlated, although the amount of shared variance was modest, $r=-.39, p<.001$. The variance inflation factor was 1.09 (anything below 5 is considered acceptable). Overall RT decreased as a function of WMC.

Our HLM analysis yielded no reliable effects for regression path times, so we present only the analyses for total time and first-pass time. The model estimates appear in Table 1.

Relative Clause Region. We found reliable effects in the relative clause region for total times. We found no reliable effects of animacy or clause type; however, we found a reliable interaction of animacy and clause type. Total time was longer in object-relative clauses with animate subjects compared to object-relative clauses with inanimate subjects. For subject-relative clauses, total time was about the same whether the subject was animate or inanimate. With respect to firstpass time, the results were similar to that for total time. We found no effects of animacy and clause type, but their interaction was reliable.

Considering only the Level-1 variables, our models produced the same pattern of results as Traxler and colleagues (2005). This is not surprising, as the model used the same Level-1 variables and the same data set. 
However, our primary interest in this analysis was the influence of WMC and overall RT on the eyemovement data. We found reliable effects of overall RT and WMC on the intercept in our analysis of total time and first-pass time in the relative clause region. Total times increased as a function of overall RT. Total fixation times decreased with increases in WMC (as in the Traxler et al., 2005, analyses). More importantly, the cross-level interaction involving overall RT, clause type, and animacy was reliable for total time, although not for first pass time. The nature of the interaction for total time is depicted in the scatter plots shown in Figure 1. Slow readers were affected more by the animacy manipulation than were fast readers, but only in object-relative sentences.

With respect to the influence of the level-2 variables, we found reliable effects of overall RT on the intercept for total time and for first-pass time in the relative clause region. Total time and first-pass time increased as a function of overall RT. We found no reliable effects of WMC. The cross-level interaction between animacy, clause type, and overall RT was reliable for total time, but not for first-pass time. We found no reliable interactions involving WMC.

Table 1

Hierarchical Linear Model - Model Estimates for Relative Clause Region

\begin{tabular}{|c|c|c|}
\hline Coefficient & Total Time & First Pass \\
\hline Intercept & $1097.44(85.72)^{* * *}$ & $739.33(43.23) * * *$ \\
\hline Clause type & $-12.13(30.15)$ & $25.25(28.00)$ \\
\hline Animacy & $4.41(27.24)$ & $18.72(17.94)$ \\
\hline Clause type x Animacy & $386.70(58.48)^{* * *}$ & $43.94(20.08)$ \\
\hline WMC & $-3.33(1.48)^{*}$ & $-1.92(1.50)^{* *}$ \\
\hline Clause type $\mathrm{x}$ WMC & $-1.033(1.95)$ & $-1.92(1.50)$ \\
\hline Animacy x WMC & $-10.70(30.09)$ & $19.05(17.97)$ \\
\hline Clause type $\mathrm{x}$ Animacy $\mathrm{x}$ WMC & $-25.30(27.95)$ & $6.38(27.10)$ \\
\hline RT & $.14(.01)^{* * *}$ & $.03(.01)^{* *}$ \\
\hline Clause type $\mathrm{x}$ RT & $-.26(.1)$ & $.00(.01)$ \\
\hline Animacy x RT & $.21(.31)$ & $-.04(.00)$ \\
\hline Clause type x Animacy x RT & $-7.54(2.94)^{*}$ & $-3.33(1.47)$ \\
\hline
\end{tabular}

Notes: Estimates are maximum likelihood. Standard errors are in parentheses.

$* \mathrm{p}<.05$

$* * \mathrm{p}<.01$

$* * * \mathrm{p}<.001$ 
Main Verb Region. The model estimates for the main-verb region appear in Table 2. We found no reliable effects of animacy or clause type on total time; however, we found a reliable interaction. Total time was longer in object-relative clauses with animate subjects compared to object-relative clauses with inanimate subjects. In sentences with subject relative clauses, total time did not differ between sentences with animate subjects and sentences with inanimate subjects. In addition, we found no reliable effects of the level-1 variables on first-pass times.

Table 2

Hierarchical Linear Model - Model Estimates for Main Verb Region.

\begin{tabular}{|c|c|c|}
\hline Coefficient & Total Time & First Pass \\
\hline Intercept & $136.94(55.73)^{* *}$ & $321.37(32.77)^{* * *}$ \\
\hline Clause type & $-20.92(15.75)$ & $6.03(12.87)$ \\
\hline Animacy & $-3.02(18.30)$ & $4.22(13.13)$ \\
\hline Clause type x Animacy & $161.61(27.32)^{* * *}$ & $24.77(20.13)$ \\
\hline WMC & $-.15(.87)$ & $-.36(.733)$ \\
\hline Clause type $\mathrm{x}$ WMC & $4.39(13.15)$ & $-3.87(18.47)$ \\
\hline Animacy x WMC & $5.83(12.94)$ & $-22.22(15.95)$ \\
\hline Clause type x Animacy x WMC & $-2.79(1.17)$ & $-1.10(.71)$ \\
\hline RT & $.06(.006)^{* * *}$ & $.02(.003)^{* * *}$ \\
\hline Clause type $\mathrm{x}$ RT & $-.26(.21)$ & $.03(.01)$ \\
\hline Animacy $x$ RT & $.14(.13)$ & $-.66(.61)$ \\
\hline Clause type x Animacy x RT & $164.14(27.67)^{* * *}$ & $25.13(20.15)$ \\
\hline
\end{tabular}

Notes: Estimates are maximum likelihood. Standard errors are in parentheses.

$* \mathrm{p}<.05$

$* * \mathrm{p}<.01$

Our chief purpose in conducting these additional analyses was to compare the previous model to an expanded model that included both WMC and overall RT as predictors of Level-1 influences on fixation times. Our results contrast with Traxler et al.'s (2005) study from which we obtained the data. In their analyses,
WMC interacted with clause-type and animacy such that low-capacity readers were affected more by the animacy manipulation in object-relative sentences than were highcapacity readers. The estimates from their model appear in Table 3. The difference between their model and the one that we constructed concerns the influence of overall 
RT. An important question is whether the model that includes overall RT is a better fit to the data than the simpler model reported by Traxler et al. We addressed this question by computing a likelihood ratio, the difference between the deviances corresponding to each model. The deviance from the model tested by Traxler et al. was 20,555.7. The deviance for the model that included overall RT (in the analysis of total time) was 20,004.2. The difference in deviances was 551.5 (2 df difference between the models). The likelihood ratio is interpreted as a chi-square, yielding a significant statistical effect, $\underline{p}<.001$. Thus, the model that included overall RT was a much better fit to the data than the simpler model that included only WMC. Moreover, our analysis suggests that the influence of WMC on the interaction of clause type and animacy was affected by its shared variance with overall RT. WMC had no influence on eye-movement fixations when overall RT was included in the model.

Table 3

Hierarchical Linear Model-Model Estimates from Traxler, Williams, Blozis, \& Morris (2005) Experiment 2

\begin{tabular}{lcc}
\hline Coefficient & Total Time & First Pass \\
\hline Intercept & $903.04(33.6)^{*}$ & $667.00(21.1)^{*}$ \\
Clause type & $22.90(24.7)$ & $35.80(19.0)$ \\
Animacy & $-3.43(24.9)$ & $-1.94(19.0)$ \\
Clause type x Animacy & $203.00(35.3)^{*}$ & $44.90(26.8)$ \\
WMC & $-5.95(2.7)^{*}$ & $-3.55(1.7)^{*}$ \\
Clause type x WMC & $3.22(2.0)$ & $.67(1.6)$ \\
Animacy x WMC & $-.42(2.0)$ & $.86(1.5)$ \\
Clause type x Animacy x WMC & $-7.11(2.9)^{*}$ & $-2.92(2.2)$
\end{tabular}

Notes: Estimates are maximum likelihood. Standard errors are in parentheses.

$* p<.05$

$* * p<.01$ 
FIGURE 1: Mean total time for subject (top) and object relative clauses (bottom) for sentences with animate and inanimate subjects, plotted against average reading time.
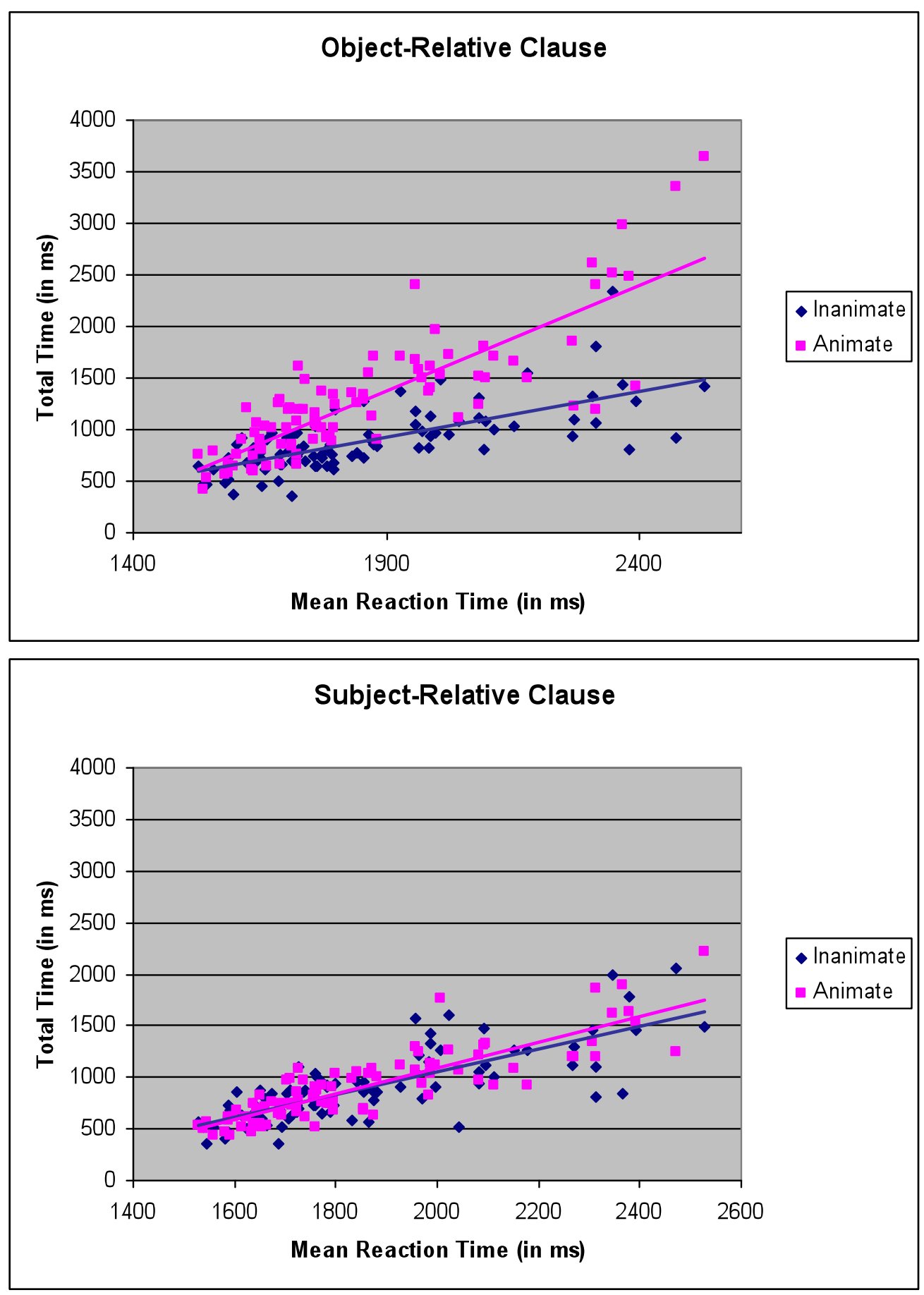


\section{Discussion}

Participants read sentences containing subjectand object-relative clauses, in which animacy of the critical nouns in the sentences provided cues either reinforcing the syntactic information or conflicting with it. When the animacy cues reinforced the correct interpretation, readers had little difficulty processing the difficult object-relative construction. Further, overall reading speed had significant cross-level interactions with animacy and clause type; slower readers were more disadvantaged than faster readers in sentences with animate subjects and object relative clauses (see Figure 1). Most important was our finding that the cross-level interactions with working-memory capacity that were reported by Traxler et al. (2005) disappeared when overall RT was included in the model. Moreover, the model was a much better fit to the data than the simpler model that included only WMC at the second level. Hence, it appears that individual variation in the influence of sentence-level variables on patterns of eyemovements is influenced more by overall reading speed than by working-memory capacity.

We are somewhat cautious in our interpretation of these results, however, for two important reasons. First, our working-memory measure and our reading speed measure shared considerable variance (almost $20 \%$ ). The variables were not correlated enough to create problems of collinearity; nonetheless, there was sufficient overlap between the two measures to make us cautious about excluding the possibility of subtle, independent effects of working memory. Second, we consider it likely that both working memory and overall reading speed serve as proxies for yet other unobserved, but nonetheless influential individual-difference variables. For example, MacDonald and Christiansen (2002) describe a view of working memory in which working-memory measures assess two factors: the quality of individuals' phonological and orthographic representations and their experience with language. In the neural network architectures that MacDonald and her colleagues advocate, there is no clear component that can be labeled as working memory. Their account could explain the relation between our individual-difference variables and how these variables influence the interaction between animacy and clause type; all of these relations may reflect the efficiency with which readers are able to access the lexicon and integrate words into their developing sentence representations. Fast readers who have more reading experience than slow readers will have more experience with the object-relative structure. Their efficiency at lexical processing and experience with the structure would make them less susceptible to the object-relative penalty than slow readers regardless of the semantic cues that are available in the sentence. Essentially, fast readers treat both sentence types as high frequency tokens. Slow readers, by virtue of less practice and exposure, rely more on cues to sentence meaning than fast readers, and so they may be especially disadvantaged when those cues are misleading, as they are when the sentence has an animate subject in the main clause and an inanimate subject in the relative clause. Considerable further research will be required, involving substantially wider varieties of individual-difference assessments, to satisfactorily determine whether this account explains results like those obtained in the current study, but our view is that it provides an entirely plausible explanation for why the model fits are better when processing speed is included.

Next, we turn to the question of how models of eye-movement control in reading might accommodate effects like those obtained here. Our view is that models of eye-movement control should not incorporate a working-memory component until there is clear evidence that the influence of working memory on eye-movements is central and not derivative from other individual differences. Hence, we focus on the influence of global processing speed on eye-movement control.

Some models have already instantiated reading speed as a group-difference factor when modeling outcomes of reading-time studies. For example, Rayner and colleagues (Rayner et al., 2006) parametrically manipulated word frequency and predictability in 
sentences that were read by younger (college age) or older (mean age $771 / 2$, range 70 to 92) participants. Processing difficulty was also manipulated; some of the sentences were presented in an easy-to-read font, whereas others were presented in old-English style font. Both groups showed frequency, predictability, and fontstyle effects, but older readers had longer overall reading times than younger readers and were affected more by frequency. Hence, the patterns of fixation durations were roughly equivalent in the younger and older groups. However, older readers were more likely to skip words than younger readers, and were more likely to re-fixate words that they had skipped. These effects were modeled in the E-Z reader architecture by incorporating a word-guessing heuristic and setting the guessing likelihood higher in the older readers than in the younger readers. Further, the authors assumed that guessing would produce errors on a fixed proportion of attempts $(10 \%)$. These parameters resulted in a very good fit between predicted and observed fixation times, wordskipping, and refixations.

In addition to age differences, there may be differences within age-groups such that some readers may rely more on a guessing strategy for identifying upcoming words than other readers. For example, Rayner and colleagues (2006) viewed willingness to skip words in older readers as being jointly influenced by reduced visual acuity, which makes specific words harder to identify from their visual features, and greater knowledge and experience, which raises readers' confidence that their guesses will be correct. Even young readers might vary along these dimensions, particularly with respect to knowledge and experience; thus, individual differences in word skipping and refixations would be expected. For example, research has shown that less-skilled readers rely more on context in word identification than do skilled readers (see Perfetti, 1985 for a review) because their word-identification skills are poorer. Thus, less-skilled readers may rely more a guessing strategy than do good readers, even though their guesses may be less accurate because they have less knowledge about words. E-Z reader has already successfully modeled variability at the group level; thus, a modest extension of the model (perhaps by using individual gamma distributions in the simulations, rather than a single overall gamma distribution) might account for the types of effects that we revealed in our analyses.

Overall speed differences might also be modeled by including individual variation in the lexical access parameter estimates in the E- $Z$ reader architecture (e.g., Reichle, Pollatsek, Fisher, \& Rayner, 1998). According to E-Z reader, saccades are planned and executed in the 250-280 ms that a reader typically spends fixating on an individual word. Planning must begin no later than about $100 \mathrm{~ms}$ after the reader begins to fixate a word (because saccades take about 150 milliseconds to plan and execute). Subtracting the approximately $50 \mathrm{~ms}$ that it takes to propagate a signal from the retina to the occipital cortex leaves very little time for lexical processing prior to the initiation of saccade planning. E$\mathrm{Z}$ reader, therefore, posits two stages of lexical access. In the first (L1 stage), readers execute a fast, approximate familiarity check. If this check yields a sufficiently high familiarity assessment, readers simultaneously undertake a second (L2) stage of lexical access and initiate saccade planning. In the L2 stage, specific properties of the word are identified that allow it to be incorporated into the developing sentence and discourse representation. One source of individual variability in fixation durations could be tied to the L1 stage of lexical access. More experienced readers or readers who have greater overall processing speed could complete the L1 stage more rapidly on average than less experienced or slower readers. However, given that the L1 stage is completed incredibly quickly by most readers most of the time (as it must if the next saccade is to be planned within the limits imposed by neural transmission), thus it seems unlikely that varying length of the L1 process will produce major variations in the fixation duration across individuals. Additionally, readers may differ in terms of their familiarity thresholds (in fact, when readers are encouraged to adopt processing strategies to deal with difficult sentences, reading times sometimes increase, rather than decrease, Traxler \& Tooley, 2008). Further, 
if individuals differ in the quality, quantity, and organization of their lexical knowledge, as research suggests they do (Perfetti, 1985), we would expect individual differences in the time that it takes to complete the L2 stage. These individual differences might be stronger on word $\mathrm{N}+1$ (during which the L2 stage on the preceding word might be completed) than on word $\mathrm{N}$, simply because there is more time available for L2 to cancel incipient saccade planning. By the same logic, we might expect greater individual variation in "spillover" effects and re-reading behavior than in eye-movement measures that index early aspects of saccade planning.

Individual differences in cognitive control or allocation of attention might also influence patterns of fixation times according to E-Z reader. In earlier versions of the model, attention-shifts from one word to the next were modeled as though they took place instantaneously. More recent versions (e.g., Reichle et al., 2009), however, incorporate more realistic, greaterthan-zero values for the attention-shift. Skilled adult readers may differ in their ability to flexibly allocate attention, and these differences could conceivably be modeled in the E-Z reader architecture by varying the attention-shift parameter across participants.

Finally, we turn to aspects of eye-movement models like SWIFT (Engbert et al., 2002; 2005) that posit simultaneous lexical processing of multiple words during reading. It appears that fewer studies have used SWIFT as a test-bed to predict individual and group differences in eye-movement behavior than EZ Reader, but this does not mean that SWIFT cannot accommodate such differences.

The SWIFT account differs from serial lexical processing accounts like E- $Z$ reader in a number of ways, but the most striking is that attentional resources and lexical access are distributed in a graded fashion across multiple words during reading, rather than being focused on a single word at a time. This account offers a rich environment in which to speculate about how differences in basic cognitive capacities could give rise to substantial variation in reading behavior. For example, in the original formulation of the SWIFT model, attention was distributed across four words, including the fixated word, one word to the left, and two words to the right. Perhaps individuals differ in the extent to which they can distribute attention this broadly. Thus, differences in the size of the attentional window might lead to variation in reading performance. In fact, substantial differences in reading behavior result when perceptual span is artificially manipulated, with the degree of disruption of reading depending on both the overall size of the perceptual window and whether foveal or parafoveal information is degraded (see, e.g., Blanchard, Pollatsek \& Rayner, 1989; Rayner, 1989; Rayner \& Balota, 1989; Rayner \& Pollatsek, 1989). Apart from the scope of distributed attention, the ability to dynamically modulate the locus of attention should also affect lexical access and saccade planning, according to SWIFT. In the SWIFT model, saccades occur when attention shifts covertly to points in the text where further lexical processing requires the greatest visual acuity (Legge's Mr. Chips model also has a similar feature; Legge, Klitz, \& Tjan, 1997). Readers who are less able to shift attention should have greater fixation times overall, as there will be a greater mismatch between the lexical processor's input requirements, the gradient of attention, and the current fixation location, compared to readers who are better able to allocate attention in the appropriate way. The autonomous saccade component of the SWIFT model, under which saccades are initiated at random intervals, mediated by inhibition from lexical processing based on foveal targets, is also a potential target for individual difference modeling. Individuals with faster overall processing speed should have a shorter random interval than individuals with slower overall processing speed. Also, readers who differ in terms of the quality of their lexical representations or who differ in the speed with which they are able to complete lexical access should differ in terms of the degree to which the autonomous saccade control mechanism is susceptible to inhibition by foveal lexical processing. In summary, the SWIFT architecture seems fully capable of 
accommodating, and formally modeling, individual variation in reading performance.

\section{Conclusions}

Our main empirical conclusion is that overall reading speed accounts for more variation in individuals' responses to syntactic and semantic manipulations in sentences containing object-relative clauses than does working-memory capacity. This contrasts with previous descriptions of the processing of this sentence type (e.g., King \& Just, 1991; Traxler et al., 2005). In the current study, greater variability in the eye-movement record was accounted for by a measure of overall reading speed than by a measure of working memory capacity. Future research that incorporates multiple measures of individual differences will be essential to our understanding of the factors that are causally central in producing variation in reading behavior and comprehension outcomes. We believe that the next generation of eye-movement control models can and should incorporate individual variation in reading performance. Such a modeling enterprise is likely to yield further testable predictions about how and why individual variation in reading occurs. 


\section{References}

Ashby, J., Rayner, K. \& Clifton, C. (2005). Eye movements of highly skilled and average readers: Differential effects of frequency and predictability. The Quarterly Journal of Experimental Psychology A: Human Experimental Psychology. 58A(6), 10651086.

Baddeley, A.D., \& Hitch, G.J. (1974). Working memory. In G.A. Bower (Ed.), The Psychology of Learning and Motivation, vol. 8, Advances in Research and Theory. San Diego, CA: Academic Press.

Blanchard, H.E., Pollatsek, A., \& Rayner, K. (1989). The acquisition of parafoveal word information in reading. Perception \& Psychophysics, 46, 85-94.

Caplan, D. \& Waters, G.S. (1990). Short-term memory and language comprehension: A critical review of the neuropsychological literature. In G. Vallar \& T. Shallice (Eds.), Neuropsychological impairments of short-term memory. (pp. 337-389). New York, NY, US: Cambridge University Press.

Caplan, D. \& Waters, G.S. (1999). Verbal working memory and sentence comprehension. Behavioral and Brain Sciences. 22, 77-126.

Clifton, C., Traxler, M.J. Mohamed, M.T., Williams, R.S., Morris, R.K., \& Rayner, K. (2003). The use of thematic role information in parsing: Syntactic processing autonomy revisited. Journal of Memory and Language. 49, 317-334.

Daneman, M. \& Carpenter, P. (1980). Individual differences in working memory and reading. Journal of Verbal Learning \& Verbal Behavior, 19, 450-466.

Engbert, R., Longtin, A., \& Kliegl, R. (2002). A dynamical model of saccade generation in reading based on spatially distributed lexical processing. Vision Research. 42, 621-636.
Engbert, R., Nuthmann, A., Richter, E.M., \& Kliegl, R. (2005). SWIFT: A Dynamical Model of Saccade Generation During Reading. Psychological Review. 112, 777-813.

Fedorenko, E., Gibson, E., \& Rohde, D. (2006). The nature of working memory capacity in sentence comprehension: Evidence against domain-specific working memory resources. Journal of Memory and Language, 54, 541-553.

Fedorenko, E., Gibson, E., \& Rohde, D. (2007). The nature of working memory in linguistic, arithmetic, and spatial integration processes. Journal of Memory and Language, 56, 246-269.

Gernsbacher, M.A. (1989). Mechanisms that improve referential access. Cognition, 32, 99-156.

Gernsbacher, M.A. (1993). Less skilled readers have less efficient suppression mechanisms. Psychological Science, 4, 294-298.

Gernsbacher, M.A. \& Faust, M.E. (1991). The mechanism of suppression: A component of general comprehension skill. Journal of Experimental Psychology: Learning, Memory, and Cognition, 17, 245-262.

Just, M. A., \& Carpenter, P . A. (1992). A capacity theory of comprehension: Individual differences in working memory. Psychological Review, 99, 122149.

Kemper, S., Crow, A., \& Kemtes, K. (2004). EyeFixation Patterns of High- and Low-Span Young and Older Adults: Down the Garden Path and Back Again. Psychology and Aging. 19, 157-170.

King, J. \& Just, M.A. (1991). Individual differences in syntactic processing: The role of working memory. Journal of Memory and Language. 30, 580-602.

Kliegl, R. Grabner, E., Rolfs, M., \& Engbert, R. (2004). Length, frequency, and predictability effects of words 
on eye movements in reading. European Journal of Cognitive Psychology. 16, 262-284.

Legge, G.E., Klitz, T.S., \& Tjan, B.S. (1997). Mr. Chips: An ideal-observer model of reading. Psychological Review, 104, 524-553.

Long, D.L., Johns, C.L., \& Morris, P.E. (2007). Comprehension Ability In Mature Readers. In M. Traxler \& M. Gernsbacher (Eds.), Handbook of Psycholinguistics (pp. 801-834). Burlington, MA: Academic Press.

MacDonald, M.C., \& Christiansen, M.H. (2002). Reassessing working memory: Comment on Just and Carpenter (1992) and Waters and Caplan (1996). Psychological Review. 109, 35-54.

Mak, W.M., Vonk, W., \& Schriefers, H. (2002). The influence of animacy on relative clause processing. Journal of Memory and Language. 47, 50-68.

Pearlmutter, N.J. \& MacDonald, M.C. (1995). Individual differences and probabilistic constraints in syntactic ambiguity resolution. Journal of Memory and Language. 34, 521-542.

Perfetti, C.A. (1985). Reading ability. New York, NY: Oxford University Press.

Preacher, K.J., Rucker, D.D., MacCallum, R.C., \& Nicewander, W.A. (2005). Use of the Extreme Groups Approach: A Critical Reexamination and New Recommendations. Psychological Methods. 10, 178-192.

Raudenbush, S.W. \& Bryk, A.S. (2001). Hierarchical Linear Models: Applications and Data Analysis Methods (2E). Thousand Oaks, CA: Sage Publications, Inc.

Rayner, K. (1989). Eye movements and the perceptual span in beginning and dyslexic readers. In C. von Euler, I. Lundberg, and G. Lennerstrand (Eds.), Brain and reading. New York, NY: Macmillian Press.
Rayner, K., \& Balota, D. A. (1989). Parafoveal preview effects and lexical access during eye fixations in reading. In W. Marslen-Wilson (Ed.), Lexical Representation and Process. Cambridge, MA: MIT Press.

Rayner, K., Chace, K.H., S.T., \& Ashby, J. (2006). Eye Movements as Reflections of Comprehension Processes in Reading. Scientific Studies of Reading. 10, 241-255.

Rayner, K., \& Pollatsek, A. (1989). The Psychology of Reading. Mahwah, NJ: Erlbaum.

Rayner K, Reichle E.D., Stroud M.J., Williams C.C., \& Pollatsek A. (2006). The effect of word frequency, word predictability, and font difficulty on the eye movements of young and older readers. Psychology and Aging, 21, 448-65.

Reichle, E., McConnell, D., \& Warren, T. (2009). Using $\mathrm{E}-\mathrm{Z}$ reader to model the effects of higher-level language processing on eye movements during reading. Psychonomic Bulletin and Review, 16, 1-21.

Reichle, E.D., Pollatsek, A., Fisher, D.L., \& Rayner, K. (1998). Toward a model of eye movement control in reading. Psychological Review. 105, 125-157.

Salthouse, T.A. (1996). The processing-speed theory of adult age differences in cognition. Psychological Review. 103, 403-428.

Traxler, M.J., \& Long, D.L. (in press). Working memory and language. In H. Pashler (Ed.), The Encyclopedia of the Mind. San Diego, CA: Sage.

Traxler, M.J., Morris, R.K., \& Seely, R.E. (2002). Processing subject and object relative clauses: Evidence from eye movements. Journal of Memory and Language. 47, 69-90. 
Journal of Eye Movement Research

$5(1): 5,1-16$.

Individual

Differences in Reading 